\title{
Is Nurse's Knowledge of DISKUS Inhaler Use Sufficient to Provide Accurate Patient Education?
}

\author{
Fatemeh Sadry ${ }^{1}$,MSN, RN;Joyce Foresman-Capuzzi ${ }^{2}, M S N$, APRN,CCNS, \\ FAEN; Mary S. Canan ${ }^{3}, \mathrm{MSN}, \mathrm{RN}, \mathrm{CNML}$; Deborah Andreson ${ }^{3}, \mathrm{MSN}, \mathrm{RN}$, \\ PCCN; Julie Gwin ${ }^{4}$, PharmD; Hortense Turner ${ }^{5}, \mathrm{MSN}, \mathrm{RN}, \mathrm{CPN}, \mathrm{CNE}$ \\ ${ }^{\prime}$ (Registered Nurse,Lankenau Hospital, Wynnewood, PA, USA) \\ ${ }^{2}$ (Clinical Nurse Educator, Lankenau Hospital, Wynnewood, PA, USA) \\ ${ }^{3}$ (Nurse Manager,Lankenau Hospital, Wynnewood, PA, USA) \\ ${ }_{4}^{4}$ (Clinical Pharmacist, Lankenau Hospital, Wynnewood, PA, USA) \\ ${ }^{5}$ (Nurse Educator, School of Nursing, Kaplan University, IA, USA)
}

\begin{abstract}
Proper handling of inhalers is essential for effective treatment of COPD and asthma patients. Nurses have a key role in educating asthma and COPD patients about correct handling of inhalers. In this study, the nurses' knowledge of handling DISKUS inhaler is evaluated before and after education which included a written instruction, an educational video, and a demonstration. For evaluation, both a questionnaire and checklist, for step-by-step demonstration, were used. Seventy percent of nurses failed the questionnaire before the training, which is reduced to ten percent after the training. All nurses handled inhaler properly after the training, while they had at least two mistakes before the training. This study proves that educating nurses about handling inhalers is needed
\end{abstract}

Keywords: inhaler, DISKUS, patient education, nurse, COPD, asthma

\section{Introduction}

One in 12 adults and one in 10 children were diagnosed with asthma in 2009, which is about 25 million or $8 \%$ of the United States population [1]. Asthma costs the US about \$3,300 per person each year, which means about $\$ 53$ billion per year [1]. Chronic Obstructive Pulmonary Disease (COPD) is the third cause of death in the United States, and it costs the US \$50 billion per year [2]. Asthma and COPD are chronic respiratory diseases that can be controlled by effective treatment. It is estimated that more than 300 million people are suffering from asthma around the word [3]. Unfortunately, the majority of patients, who are being treated with Dry Powder Inhalers (DPIs), do not use their inhalers correctly [4].

There is a need for proper educating health care professionals and patients about correct handling of dry powder inhalers. Dry Powder Inhaler (DPI) is a medicine delivery device that delivers medication in the form of dry powder. The dry powder inhalers were introduced to medical practice in the early 1970s. DPIs can deliver a single dose or multiple doses. DISKUS is a DPI that can deliver multiple doses.

The misuse of respiratory inhalers in hospitalized patients with asthma and COPD has been studied by Press et al [5]. These researchers designed a cross-sectional and pre/post intervention study at two hospitals. One-hundred COPD and asthma patients participatedin the research. They used both Metered Dose Inhaler (MDI) and DISKUS for their research. The result showed that $86 \%$ of patients misused MDI and $71 \%$ misused DISKUS. This study showed that misuse of respiratory inhaler is common; however, this problem is easily correctable in hospitalized patients. Hospitals should assess the knowledge of patients about using inhaler and educate them when it is needed.

Basheti et al used patient-centered educational tools including novel Inhaler Technique Labels to study the relation between lack of instruction, age, and increased risk of hospitalization, emergency room visits, and poor disease control [6]. They used 31 pharmacists, who received a brief workshop education. Active group pharmacists were trained to assess and teach dry powder inhaler technique, using patient-centered educational tools including novel Inhaler Technique Labels. Interventions were delivered to ninety-seven patients at four visits over six months. At baseline, patients demonstrated poor inhaler technique. After six months, the improvement in inhaler technique score was significantly greater in the active group.

Khassawneh et al studied the patients' mistakes when they use different DPI and MDI devices [7]. They used four different inhaler devices in the study, the MDI, DISKUS, Turbuhaler, and Aerolizer. The researchers observed each step of the inhalation technique with a placebo device. An appropriate form, which included demographics, and a checklist of the essential steps were completed for each device. A total of 300 patients completed the evaluation interview with the mean age of forty-eight years. Approximately half of the patients were simultaneously using two or more inhaler devices. The MDI device was incorrectly handled by 
$75 \%$, the Aerolizer by $17 \%$, the DISKUS by $7 \%$, and the Turbuhaler by $43 \%$. The result showed that patients had less mishandling with DISKUS inhaler.

Rootmensen et al studied the effect of instruction and handling multiple inhaling devices on patients' mistakes [8]. This study includes 156 patients that performed 204 inhalation demonstrations. The mean age of the participants was sixty-one years, $42 \%$ were female, $58 \%$ were diagnosed with COPD, and 44 patients $(28 \%)$ used multiple types of inhaler devices. In this study, $40 \%$ of all asthma and COPD patients made at least one essential mistake in their inhalation technique. Patients who never received inhalation instruction and patients who used more than one inhaler device made significantly more mistakes.

Melani, et al. [9] studied the relationship between inhaler misuse with older age and lower schooling. They considered 1664 adult patients (mean age sixty-two years) affected mostly by COPD (52\%) and asthma (48\%) in their research. Respectively, 843 and 1113 patients were using MDIs and DPIs at home. They used questionnaire and observation, which was recorded, to collect data. They found that there is a relation between inhaler misuse and age, lower schooling, and lack of instruction received for inhaler technique by health caregivers. The inhaler misuse was associated with increased risk of hospitalization, emergency room visits, courses of oral steroids and antimicrobials, and poor disease control.

As per the literature, a low percentage of healthcare providers can demonstrate use of inhaler correctly and free of error [10-11]. Most of researchers studied the patient education and knowledge on proper handling of inhalers [8-9]. However, the first step to educate patients about the correct use of an inhaler is to educate the healthcare providers who are teaching these patients. Registered nurses have an important role in administering and education the patients in the clinics and hospitals. However, limited research is published about nurses' knowledge of handling PDIs [11].

In order to get effective treatment from DISKUS, accurate handling of this devise is vital. There are ten steps in using this inhaler correctly; lack of one of these steps prevents complete delivery of the medication. Patients who are using DISKUS should be educated by healthcare providers. Therefore, there is a need for educating registered nurses and healthcare personnel who administer inhaler devices. Only one of the previous studies focused on educating registered nurses in hospital settings, while nurses have essential roles in teaching the patients [11]. So,there is still an unanswered question about nurses' knowledge: "are registered nursesin the hospital settings knowledgeable about accurate handling of DISKUS?"

\section{Research Method}

In order to answer above question, the knowledge of twenty registered nurses who work in a telemetry unit were evaluated. The participant nurses had a wide range of nursing experience, from one year to 25 years. This study contains three phases: Phase one included pre-training knowledge assessment of registered nurses regarding handling of DISKUS inhaler. The second phase is the training session, which included one on one education by demonstration, written material, and educational video. The third phase is post-training assessment of nurses, who received the training session, immediately after training. It took about 15 minutes for each nurse to complete all assessments and trainings.

\section{Phase 1: Pre-training knowledge and technique assessment}

Verbal consent was achieved from each participant before starting the questionnaire. The questionnaire includedfive multiple choice questions that covered general knowledge about DISKUS inhaler handling. After all participants completed the pre-training questionnaire, each participant was asked to demonstrate step by step DISKUS handling. A checklist was usedto assess their inhaler handling technique. There are ten steps in DISKUS inhaler handling. When a nurse demonstrated all steps correctly, she/he would pass the demonstration. The demonstration DISKUS did not contain any medication, as they were made just for educational purposes.

\section{Phase 2: Training session}

In this session, a one-on-one training was provided for each registered nurse. Three different educational methods were used for this training, including written step by step instructions for DISKUS handling. Then, the step by step DISKUS inhaler handling was demonstrated for each participant. Finally, a training video about step by step DISKUS handling was used.

\section{Phase 3: Post-training knowledge and technique assessment}

After completing the training, nurses' knowledge was assessed immediately. In this phase, each participant completed the same questionnaire that they completed in the pre-training. Then, they were asked to demonstrate the correct steps for using the DISKUS inhaler.

\section{Results And Discussion}

The criteria to successfully passing the assessments were $80 \%$ correct answers on the questionnaire and $100 \%$ correct steps on the demonstration. As it is shown in Figure 1a, $70 \%$ of nurses failed the questionnaire, which was related to general information about this inhaler. After the training, the percentage of nurses who passed the questionnaire increased from $30 \%$ to $90 \%$ (see Figure 1b). The result obtained for step by step pre- 
training checklist showed that all nurses missed at least two steps. It means, $100 \%$ of nurses failed step by step training. However, after the training, all nurses passed proper demonstration of DISKUS inhaler. This results shows that although, the training time was short, it was effective.

As it is shown in Figure 1b, the result of assessing post-training questionnaire was not one hundred percent, which was related to the training time. All nurses participated in this research during their working hours, so the training session hadto be very quick and short. Arranging similar training as a separate workshop outside of nurses' working time would make it more efficient.

This project proved lack of knowledge of handling DISKUS inhaler in registered nurses. As per literature, other healthcare providers, such as pharmacists and physicians, have lack of knowledge too [12]. Training of healthcare providersis very crucial in proper administering of inhalers, such as DISKUS. It is suggested that all hospitals and healthcare systems provide educational sessions/workshops for their nurses and other health care providers. Additionally, pharmacies should provide step by step checklists for handling inhalers, such asDISKUS.

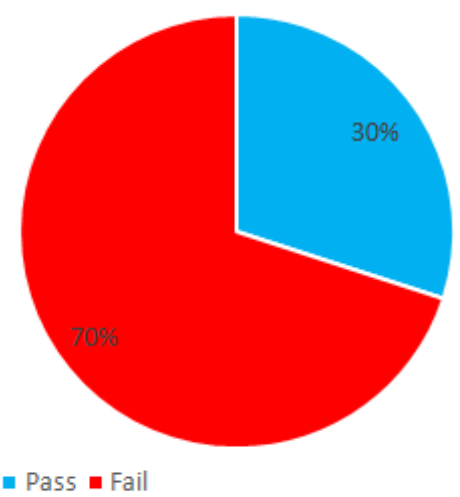

a) Before the training

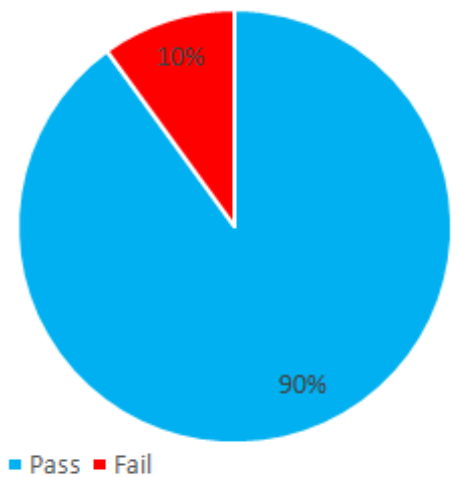

b) After the training

Figure 1: Assessment of nurses' knowledge of DISKUS inhaler, using a questionnaire.

\section{Summary And Conclusion}

Twenty nurses were participated in this research were their knowledge of handling DISKUS inhaler before and after education were assessed. Both a questionnaire and step by step demonstration were used for evaluation. A three-step process (written checklist, educational video, and demonstration) was used for education. Thirty percent of nurses passed the questionnaire before education, improving to $90 \%$ post education. All nurses failed in the step by step handling of inhaler prior to education with $100 \%$ of nurses successfully completing the hand-on portion after education, demonstrating not only that cognitive education is vital to learning, but the psycho-motor component is essential to improved outcomes.

\section{Acknowledgements}

Authors appreciate Main Line Health System for supporting this research, GlaxoSmithKline Co. for providing demonstration DISKUS devices, and all nurses who voluntarily participated in this study. Authors also appreciate Dr. Reza Masoodi for his help in analyzing the results and preparing the manuscript.

\section{References}

[1]. American Academy of Allergy Asthma \& Immunology. (2015). Asthma Statistics, United States. Retrieved from http://www.aaaai.org/about-the-aaaai/newsroom/asthma-statistics.aspx

[2]. Burks, A. W. (2012). Allergies, asthma, and respiratory disease affect many. This report will give you the tools to fight back. Retrieved from https://www.aaaai.org/Aaaai/media/MediaLibrary/PDF\%20Documents/Announcements/USAToday_RespiratoryHealth.pdf

[3]. Osman, A., Hassan, I. S. A., \& Ibrahim, M. I. M. (2012). Are Sudanese community pharmacists capable to prescribe and demonstrate asthma inhaler devices to patrons? A mystery patient study. Pharm Pract, 10(2), 110-115.

[4]. Lavorini, F., Magnan, A., Dubus, J. C., Voshaar, T., Corbetta, L., Broeders, M., \&Sanchis, J. (2008). Effect of incorrect use of dry powder inhalers on management of patients with asthma and COPD. Respiratory Medicine, 102(4), 593-604.

[5]. Press, V. G., Arora, V. M., Shah, L. M., Lewis, S. L., Ivy, K., Charbeneau, J., ... \& Krishnan, J. A. (2011). Misuse of respiratory inhalers in hospitalized patients with asthma or COPD. Journal of general internal medicine, 26(6), 635-642.

[6]. Basheti, I. A., Armour, C. L., Bosnic-Anticevich, S. Z., \&Reddel, H. K. (2008). Evaluation of a novel educational strategy, including inhaler-based reminder labels, to improve asthma inhaler technique. Patient education and counseling, 72(1), 26-33.

[7]. Khassawneh, B. Y., Al-Ali, M. K., Alzoubi, K. H., Batarseh, M. Z., Al-Safi, S. A., Sharara, A. M., \&Alnasr, H. M. (2008). Handling of inhaler devices in actual pulmonary practice: metered-dose inhaler versus dry powder inhalers. Respiratory care, 53(3), 324-328. 
[8]. Rootmensen, G. N., van Keimpema, A. R., Jansen, H. M., \& de Haan, R. J. (2010). Predictors of incorrect inhalation technique in patients with asthma or COPD: a study using a validated videotaped scoring method. Journal of aerosol medicine and pulmonary drug delivery, 23(5), 323-328.

[9]. Melani, A. S., Bonavia, M., Cilenti, V., Cinti, C., Lodi, M., Martucci, P., ..\&Neri, M. (2011). Inhaler mishandling remains common in real life and is associated with reduced disease control. Respiratory medicine, 105(6), 930-938.

[10]. Basheti, Iman A., et al. "Inhaler technique training and health-care professionals: effective long-term solution for a current problem." Respiratory care 59.11 (2014): 1716-1725.

[11]. De Tratto, K., Gomez, C., Ryan, C. J., Bracken, N., Steffen, A., \& Corbridge, S. J. (2014). Nurses' knowledge of inhaler technique in the inpatient hospital setting. Clinical Nurse Specialist, 28(3), 156-160.

[12]. Plaza, V., Sanchis, J., Roura, P., Molina, J., Calle, M., Quirce, S., ...\&Murio, C. (2012). Physicians' knowledge of inhaler devices and inhalation techniques remains poor in Spain. Journal of aerosol medicine and pulmonary drug delivery, 25(1), 16-22. 